\title{
Editorial
}

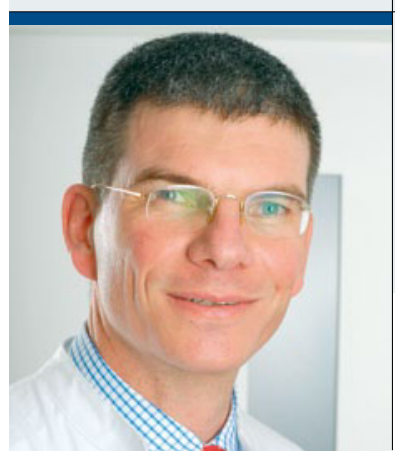

„Die völlig unzureichende Honorierung der Spermiogramme

ist ein berufspolitisches Problem, ändert aber nichts an der

Notwendigkeit, Qualität abzuliefern."

Prof. Dr. med. Frank-Michael Köhn

klinischer Androloge, München

\section{Neue Herausforderungen in der Andrologie}

n den letzten Monaten haben sich zwei neue Herausforderungen für die Andrologie herauskristallisiert: _ Mit Erscheinen des neuen WHO-Laborhandbuches sind 2010 neue Referenzwerte für die Variablen des Spermiogramms wirksam geworden, die sich teilweise erheblich von denen der Vorgänger-Auflage von 1999 unterscheiden und auf einer anderen Datenbasis beruhen.

_ Die Richtlinien der Bundesärztekammer für die Qualitätssicherung laboratoriumsmedizinischer Untersuchungen wurden mit Wirksamkeit ab dem 1.1.2011 um einen Abschnitt Ejakulatuntersuchungen erweitert, der die qualitätssichernden Maßnahmen bei der Erstellung eines Spermiogramms präzisiert und ab 1.1.2013 verbindlich macht.

Veränderungen führen manchmal zu Unruhe und Ängsten, die aber nicht immer eine reale Grundlage haben müssen. Natürlich besteht die Sorge vor ausufernder Dokumentation und zunehmendem Zeitaufwand für die gleiche Leistung bei unveränderter Honorierung. Es wird aber wesentlich darauf ankommen, wie andrologisch tätige Ärzte mit diesen Herausforderungen umgehen.

Die Kritik mancher Kollegen an den zum Teil dramatisch veränderten Referenzwerten für Spermienkonzentration, Motilität und Morphologie sollte nicht unberücksichtigt lassen, dass die Weltgesundheitsorganisation selbst auf die Limitierungen der Referenzwerte hingewiesen hat. Es wird ausdrücklich erwähnt, dass eine Unterschreitung der Werte nicht automatisch einen Verlust der Fertilität bedeutet und das Spermiogramm im Zusammenhang mit klinischen Parametern beurteilt werden muss. Hierin liegt eine Stärkung der Stellung der Andrologen, da nur sie diese Symbiose von Labor und Klinik herbeiführen können.
Auch die Forderung nach Qualitätssicherung, wie sie in den Richtlinien der Bundesärztekammer zum Tragen kommt, hat ihre Berechtigung. Die völlig unzureichende Honorierung der Spermiogramme ist ein berufspolitisches Problem, ändert aber nichts an der Notwendigkeit, Qualität abzuliefern. Unverständlich bleibt aber die Forderung nach Qualität und evidenzbasiertem Vorgehen in einem Bereich, wenn gleichzeitig Kostenträger im Gesundheitssystem vollständig ungeprüfte diagnostische und therapeutische Prozedere im heilpraktischen Sektor übernehmen.

Eine Konsequenz der Veränderungen in der Andrologie sollte aber trotz aller Ärgernisse nicht gezogen werden: der Rückzug aus der „konservativen“ Andrologie. Resultat wäre der Verlust dieses Betätigungsfeldes an die Labormedizin. Es gilt vielmehr, die oben genannten Richtlinien der Bundesärztekammer nicht nur zu befolgen, sondern auch darauf hinzuweisen, dass der Gemeinsame Bundesausschuss der Ärzte und Krankenkassen in seinen Richtlinien über ärztliche Maßnahmen zur künstlichen Befruchtung eine Einbindung von Andrologen in Diagnostik und Therapie im Rahmen der assistierten Reproduktion fordert. Es besteht somit weniger Anlass zur Sorge als der Aufruf, die Interessen der Andrologie mit Nachdruck zu verfolgen.

Ihr

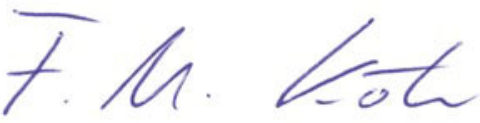

